\title{
Synthesis and characterization of surface-modified and organic- functionalized MCM-41 type ordered mesoporous materials
}

\author{
S C LAHA, P MUKHERJEE and R KUMAR* \\ Catalysis Division, National Chemical Laboratory, Pune 411008 , India
}

\begin{abstract}
A new and efficient method for the preparation of MCM-41 type ordered mesoporous phases using phosphate as promoter under reflux conditions is reported. The various mesoporous materials studied were: silica (Si-MCM-41), alumino-silicate (Al-MCM-41), and titanium-silicate (Ti-MCM-41). Our concept of promoter-assisted synthesis of zeolites and related microporous materials was found to be applicable in the synthesis of ordered mesoporous materials as well. The addition of small catalytic quantity of phosphate ions $\left(\mathrm{PO}_{4}^{3-}\right)$, used as promoters, significantly reduced the synthesis time (by a factor of 3-4) of all these mesoporous materials. The synthesis of new MCM-41 type organic-inorganic composite materials with unique properties is also reported.
\end{abstract}

Keywords. MCM-41; mesoporous materials; phosphate; promoter; synthesis.

\section{Introduction}

The discovery of ordered mesoporous materials, such as MCM-41 (Kresge et al 1992), having hexagonal arrangement of unidimensional pores of 2-10 nm diameter, has not only enlarged the domain of molecular sieves but also advanced the synthesis of new materials in general. These mesoporous materials are of interest to a broad range of areas like selective separation, catalysis, support, carriers, chemical sensors etc (Corma 1997). New applications of these interesting materials are expected to grow in the near future.

Generally, these ordered mesoporous materials are synthesized hydrothermally in an autoclave under autogeneous pressure using a surfactant like cetyltrimethylammonium cation. The synthesis of mesoporous metallosilicate MCM-41 type materials can also be carried out using a low temperature reflux method under atmospheric pressure (Carvalho et al 1997). This method was found to be particularly useful for preparing stable and catalytically-active redox metal ions incorporated MCM-41 type materials.

Recently, it has been observed (Kumar et al 1996, 1998) that the addition of small amount of certain oxyanions such as phosphate, perchlorate, arsenate, chlorate, bromate etc used as promoters, to the synthesis precursor mixture of zeolite and related silica-based molecular sieves, reduces their crystallization time considerably. This concept of promoter was found to be quite general in the synthesis of a variety of silica-based microporous (8-, 10- and 12-membered ring pore

*Author for correspondence openings) as well as mesoporous molecular sieves (Mukherjee et al 1998).

In this paper we report the promoter-assisted synthesis of pure silica, aluminum and titanium-silicate analogues of MCM-41 type mesoporous materials. The synthesis of organic functionalized MCM-41 materials and their potential uses in catalysis and carrier for nanoclusters are also discussed.

\section{Experimental}

\subsection{Synthesis}

The syntheses of Si-MCM-41, Al-MCM-41, Ti-MCM-41 and organo-functionalized MCM-41 samples were carried out as follows. For the synthesis of Si-MCM-4l in the absence of promoter (sample A, Si-MCM-41-S), a mixture of two types of silica sources: (i) sodium metasilicate, $\mathrm{Na}_{2} \mathrm{SiO}_{3}$ (s.d. fine chem., India), and (ii) amorphous silica (Areosil) were employed, where the ratio of $\mathrm{Na}_{2} \mathrm{SiO}_{3} / \mathrm{SiO}_{2}$ was $0.124: 1$. Silica sources were suspended in aqueous solution of TMAOH (25\%, Aldrich). This suspension was then combined with a solution of cetyltrimethylammonium bromide (CTMABr, Aldrich) in water. The initial gel composition was $1.0 \mathrm{SiO}_{2}: 0.22 \mathrm{NaOH}: 0.1$ $\mathrm{NaCl}: 0.1$ TMAOH : 0.21 CTMABr: $125 \quad \mathrm{H}_{2} \mathrm{O}$. For preparing the Si-MCM-41 using promoter (sample $\mathrm{B}$, $\mathrm{Si}-\mathrm{MCM}-41-\mathrm{P}$ ), a solution of $\mathrm{NaH}_{2} \mathrm{PO}_{4}$ (s.d. fine chem., India) in water was added in the final gel, using the same above-mentioned procedure. To maintain the $\mathrm{Si} / \mathrm{Na}$ molar ratio in the gel of Si-MCM-41-S and Si-MCM41-P samples the same, a required amount of $\mathrm{NaCl}$ was added to the former sample. In Si-MCM-41-P sample, the molar gel composition was $1.0 \mathrm{SiO}_{2}: 0.22 \mathrm{NaOH}: 0.1$ $\mathrm{NaH}_{2} \mathrm{PO}_{4}: 0.1$ TMAOH : 0.21 CTMABr : $125 \mathrm{H}_{2} \mathrm{O}$. 
2.1a Synthesis of organo-functionalized Si-MCM-4I mesoporous materials: For preparing organic functionalized MCM-41 (samples G and H), the 3-X-propyltrimethoxysilane (XPTS) was used along with TEOS (in $1: 2 \cdot 5-4$ ratio) as silica source $\left(\mathrm{X}=\mathrm{NH}_{2}\right.$ or $\left.\mathrm{SH}\right)$.

The gel composition of the materials was 1.0 XPTS : 2.5 TEOS : 0.42 CTMABr : 0.96 NaOH : 272 $\mathrm{H}_{2} \mathrm{O}: 66 \mathrm{MeOH}$. To slow down the rate of hydrolysis of XPTS, methanol was added to the mixture. The reaction mixture was put in an autoclave and kept at $95^{\circ} \mathrm{C}$ for $36 \mathrm{~h}$. The product was then filtered and washed several times with distilled water and methanol. At first it was dried in air and then under vacuum. The surfactant was removed by the extraction with a mixture containing $68 \mathrm{~g}$ methanol, $1.9 \mathrm{~g} \mathrm{HCl}(35.4 \%)$ and $2.2 \mathrm{~g}$ water per $\mathrm{g}$ of the solid MCM-41 materials under reflux conditions.

The synthesis of Al-MCM-41 samples was also carried out in the same way as that of $\mathrm{Si}-\mathrm{MCM}-41$ samples except that the source of $\mathrm{Al}_{2} \mathrm{O}_{3}\left(\mathrm{Al}_{2} \mathrm{SO}_{4}: 16 \mathrm{H}_{2} \mathrm{O}\right.$, s.d. fine chem., India) was also added. The molar ratio $\mathrm{SiO}_{2} / \mathrm{Al}_{2} \mathrm{O}_{3}$ was 40 . Remaining gel composition and the promoter used $\left(\mathrm{NaH}_{2} \mathrm{PO}_{4}\right)$ were the same as those for the Si-MCM-41 samples.

In a typical synthesis of Ti-MCM-41-S (standard), the similar procedure and gel composition was used as given above for Si-MCM-41 samples, except that a solution of tetrabutyl titanate (TNBT, Aldrich) in isopropanol was added, adapting from literature (Thangaraj et al 1991) for TS-1. The molar gel composition was $1.0 \mathrm{SiO}_{2}: 0.018 \mathrm{TiO}_{2}: 0.3 \mathrm{TMAOH}: 0.21 \mathrm{CTMABr}$ : $125 \mathrm{H}_{2} \mathrm{O}$. For preparing Ti-MCM-41-P sample, $\mathrm{H}_{3} \mathrm{PO}_{4}$ ( $85 \%$, s.d. fine chem., India) was also added in the final gel as prepared for Ti-MCM-41-S sample. The $\mathrm{SiO}_{2}: \mathrm{H}_{3} \mathrm{PO}_{4}$ molar ratio was $1: 0 \cdot 1$. The rest of the gel composition was same as that of the Ti-MCM-4l-S sample.

The synthesis of the samples (except organic functionalized MCM-41 samples) was carried out under reflux in a glass flask. After refluxing the gel for the required time, the solid products were collected by filtration, washed several times with distilled water, and dried at $373 \mathrm{~K}$ and calcined at $500^{\circ} \mathrm{C}$ for $12 \mathrm{~h}$ in flowing air.

\subsection{Characterization}

The samples were characterized by X-ray diffraction (Regaku D Max III, VC diffractometer), scanning electron microscopy (SEM, Jeol JSM 5200), Fourier transform infrared spectroscopy (FTIR), Perkin Elmer 1600, Nujol on $\mathrm{KBr}$ disc technique, chemical analysis, pore size measurement, and Braunauer-Emmett-Teller (BET) surface area (Omnisorp CX-100).

\section{Results and discussion}

Table 1 depicts the synthesis time $(h)$ for the various samples (A-F). It is evident that there is a considerable reduction in the synthesis time required for all the samples prepared in the presence of phosphate promoter (samples B, D, F) compared to those prepared in the absence of promoter (samples A, C, E). However, the time taken for the synthesis of various MCM-41 materials followed the trend: Si-MCM-41 $\cong$ Ti-MCM-41, 〈AlMCM-41 both in the presence as well as in the absence of promoter. Based on liquid state NMR studies on the synthesis mixture of silicalite-1 (Kumar et al 1998), it was found that the presence of promoter oxyanions enhances the condensation process of silicate-template composite species, mainly involving $\mathrm{Q}^{4}$ silicate entities. High polarizing ability of the promoter anions probably polarizes water spheres formed around silicate and template molecules towards itself and thereby facilitate the overlapping of silicate and template moieties resulting in the formation of composites species. It is reasonable to envisage a similar role of the phosphate ions in the synthesis of MCM-41 type of mesoporous materials also.

XRD of all the samples exhibited weak 110,200 and $210 \mathrm{hkl}$ lines along with main 100 reflection indicating high degree of hexagonal ordering. The Si-MCM-41 (sample A) exhibited worm-like morphology having average particle size $2 \times 10 \mu \mathrm{m}$. Although, the particle shape of both the samples (A and B) was quite similar, there was a significant reduction in the particle size of sample B (prepared in the presence of promoter) compared to that of sample A (prepared in the absence of promoter). The same was found to be true for other samples as well. This is quite expected because of significant increase in the ordering rate in the presence of promoter at the initial stages of the synthesis of MCM-41 type mesoporous materials.

In table 1 the surface area of these mesoporous materials is given. High BET surface area of all the samples indicates that all the samples have highly ordered mesoporous structure. Sample B (Si-MC̣M-41-P) exhibited higher surface area $\left(1480 \mathrm{~m}^{2} \mathrm{~g}^{-1}\right)$ compared to that of sample A $\left(1296 \mathrm{~m}^{2} \mathrm{~g}^{-1}\right)$, which may be attributed to the smaller particle size of the former. Almost similar trend is also observed for samples $\mathrm{C}$ and $\mathrm{D}$ (Al-MCM-41 materials) as well as samples $\mathrm{E}$ and $\mathrm{F}$ (Ti-MCM-41). The adsorption isotherms of nitrogen and the infrared

Table 1. Characterization of MCM-41 type mesoporous materials.

\begin{tabular}{lccc}
\hline & $\begin{array}{c}\text { Synthesis } \\
\text { time } \\
(\mathrm{h})\end{array}$ & $\begin{array}{c}\text { Surface } \\
\text { area } \\
\left(\mathrm{m}^{2} \mathrm{~g}^{-1}\right)\end{array}$ & $\begin{array}{c}\text { Average } \\
\text { particle size } \\
(\mu \mathrm{m})\end{array}$ \\
\hline Sample & 16 & 1296 & $2 \times 10$ \\
A (Si-MCM-41-S) & 6 & 1480 & $0.5 \times 0.8$ \\
B (Si-MCM-41-P) & 64 & 930 & n.d. \\
C (Al-MCM-41-S) & 24 & 1030 & $0.8 \times 1.2$ \\
D (Al-MCM-41-P) & 12 & 1245 & $2 \times 10$ \\
E (Ti-MCM-41-S) & 16 & 1050 & $0.4 \times 0.6$ \\
F (Ti-MCM-41-P) & 8 & & \\
\hline
\end{tabular}


Table 2. Chemical analysis (wt \%), surface area and pore size of organo-functionalized MCM-41 samples.

\begin{tabular}{lcccccc}
\hline Samples & C & N & S & H & $\begin{array}{c}\text { Pore size } \\
(\AA)\end{array}$ & $\begin{array}{c}\text { Surface area, } \\
\left(\mathrm{m}^{2} / \mathrm{g}\right)\end{array}$ \\
\hline $\mathrm{C}_{3} \mathrm{H}_{7} \mathrm{SH}-\mathrm{MCM}-41(\mathrm{G})$ & 13.25 & - & 8.56 & 2.72 & 40 & 830 \\
$\mathrm{C}_{3} \mathrm{H}_{7} \mathrm{NH}_{2}-\mathrm{MCM}-41(\mathrm{H})$ & 11.63 & 4.33 & - & 4.26 & 24 & 550 \\
\hline
\end{tabular}

spectra (1300-400 $\mathrm{cm}^{-1}$ range) of MCM-41 samples prepared in the presence of promoter (samples B, D and F) exhibited characteristic patterns of ordered MCM-41 type mesoporous materials.

\subsection{Organo-functionalized (organo-inorganic) MCM-41 materials}

The elemental analyses of the samples $\mathrm{G}$ (thiol) and $\mathrm{H}$ (amine) are given in table 2. The adsorption pattern of the samples indicated that they are neither of pure type-I, typical of microporous solids, nor of pure type-IV, typical of mesoporous solids. Instead they had slopes in the $p / p_{0}$, range $0 \cdot 1$. This slope may arise due to capillary condensation of the adsorbate inside the mesopores of the material. The pore size and BET surface area of the sample $G$ were $40 \AA$ and $836 \mathrm{~m}^{2} \mathrm{~g}^{-1}$, respectively, indicating the presence of mesoporous structure. However, the surface area observed in the case of propane aminefunctionalized material (sample $\mathrm{H}$ ) is considerably less $\left(550 \mathrm{mg}^{-1}\right)$ than that of the propane thiol-functionalized MCM-41 material (sample G). Its pore size maxima appeared almost half $(24 \AA)$ of the thiol-functionalized materials. Thus, this is an interesting method for controlling the pore size and make new materials with unique properties. Further, these materials are potentially important molecular sieves because of their high surface area and modifiable pore size. The prominent peaks of ${ }^{13} \mathrm{C}$ CP MAS spectrum (sample G) arise as a result of different types of organo-sulfur groups. The most prominent peak arises from 3-marcaptopropyl groups with resonances at $27.2 \mathrm{ppm}$ for $\mathrm{Cl}$ carbon atom adjacent to SH-group and $\mathrm{C} 2$ carbon atom, and $11.8 \mathrm{ppm}$ for $\mathrm{C} 3$ carbon atom. Another minor intensity peak appears at $22.3 \mathrm{ppm}$, assigned to $\mathrm{C} 1$ and $\mathrm{C} 2$ carbon atoms of the dipropylsulphide (thioether) which is formed due to the oxidation product between adjacent thiol groups (Lim et al 1998). The C3 carbon atom of disulphide produces an unresolved shoulder slightly down field of the C3 thiol carbon. Two minor additional peaks at 50.2 and 36.5 appeared due to residual methanol used for extraction (figure 1).

There is tremendous scope in this area as the functional groups can be used as ligand for various metal ions or even metal complexes, including with a chiral moiety.
PSH-CN ns $=1600$ at $2.424 \mathrm{kHz}$

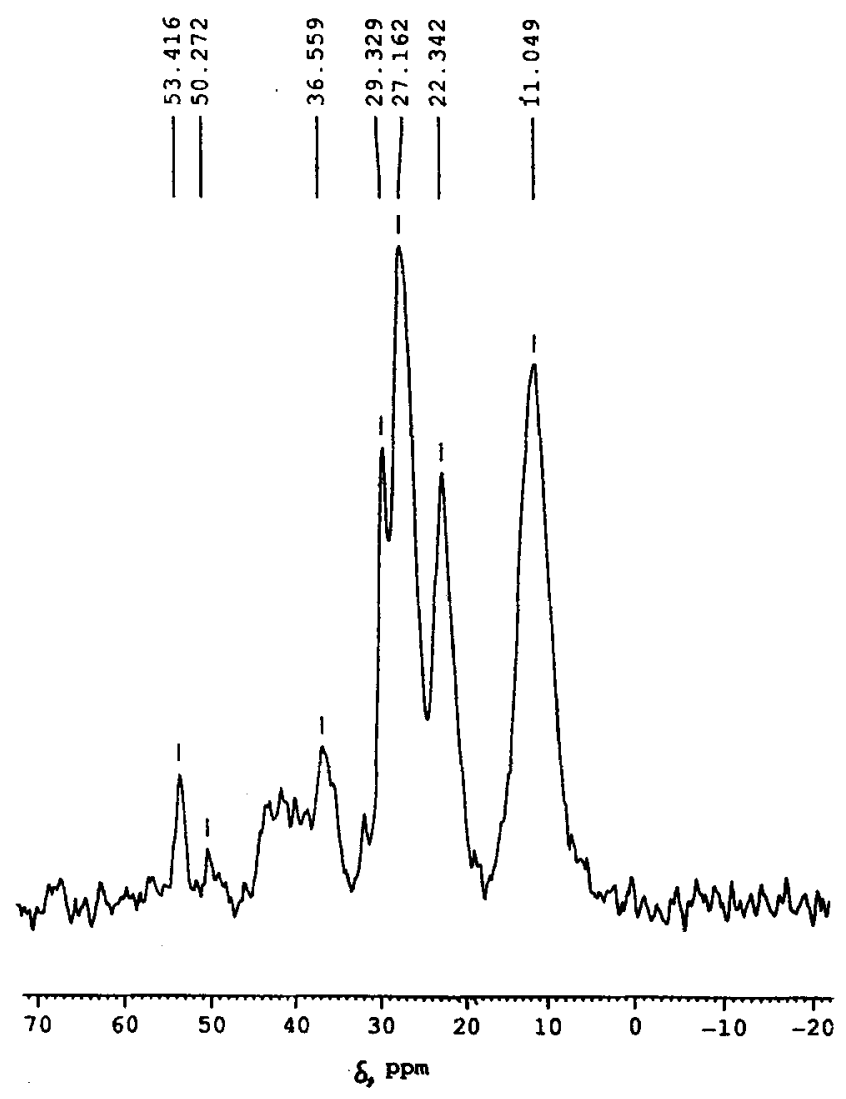

Figure 1. ${ }^{13} \mathrm{C}$ CP MAS spectrum (Brucker MSL 300, TMS as standard) of organic functionalized, propyl thio-MCM-41 (sample $\mathrm{G}$ ) after the extraction of the surfactant.

Such composite solid catalyst materials may exhibit interesting catalytic property. Even nanoclusters of noble metals like $\mathrm{Ag}, \mathrm{Au}$, etc are encapsulated in these organoinorganic MCM-41 type materials (Mukherjee et al, unpublished results).

\section{Conclusions}

In this paper we have shown that the addition of small amount of phosphate ions (promoter) to the synthesis mixture significantly reduces the synthesis time of MCM- 
41 type mesoporous materials. Thus the concept of promoter oxyanions is not only useful to zeolites and related microporous materials (Kumar et al 1996, 1998) but is shown to be useful in the case of mesoporous materials as well. The materials synthesized in the presence of promoter exhibited smaller particles vis-á-vis those synthesized in the absence of the promoter ions. The synthesis and characterization of new organic functionalized (organo-inorganic composite) MCM-41 type materials has also been discussed.

\section{Acknowledgements}

Two of us (SCL and PM) are grateful to CSIR, New Delhi, for the Research Fellowships.

\section{References}

Carvalho W A, Varaldo P B, Wallau M and Schuchardt U 1997 Zeolites 18408

Corma A 1997 Chem. Rev. 972373

Kumar R, Bhaumik A, Ahedi R K and Ganapathy S 1996 Nature 381298

Kumar R, Mukherjee P, Pandey R K, Rajmohanan P and Bhaumik A 1998 Microporous \& Mesoporous Mater. 2223

Kresge C T, Leonowicz M E, Roth W J, Vertuli J C and Beck J S 1992 Nature 359710

Lim H M, Blanford F C and Stein A 1998 Chem. Mater. 10 46

Mukherjee P, Kumar R and Schuchardt U 1998 Stud. Surf. Sci. Catal. 117351

Thangaraj A, Kumar R, Mirajkar S P and Ratnasamy P 1991 J. Catal. 1311 